\title{
CORRECTION
}

\section{LOCALLY LATTICE SAMPLING DESIGNS FOR ISOTROPIC RANDOM FIELDS}

\author{
By Michael L. Stein
}

Annals of Statistics (1995) 23 1991-2012

In the limiting variance given in the statement of Theorem $1, \phi(x)$ should be raised to the $-p / 2$ power, not the $-p$ power as is incorrectly given in the paper. More specifically, the displayed equation in Theorem 1 should read

$$
\begin{aligned}
& g\left(N_{n}^{1 / 2}\right)^{-1} \operatorname{var}\left(\int_{G} v(x) Z(x) d x-\hat{Z}_{n}\right) \\
& \quad \rightarrow(2 \pi)^{2-p} \int_{G} v(x)^{2} \phi(x)^{-p / 2} d x \sum_{K}{ }^{\prime}|M K|^{-p} .
\end{aligned}
$$

Similarly, on pages 2000 and 2001, there are several instances where $\int_{G} v(x)^{2} \phi(x)^{-p} d x$ should be replaced by $\int_{G} v(x)^{2} \phi(x)^{-p / 2} d x$ and in (3.11), $\left\{r_{J}^{-1} \int_{R_{J}} \phi(x) d x\right\}^{p}$ should be $\left\{r_{J}^{-1} \int_{R_{J}} \phi(x) d x\right\}^{-p / 2}$. As a consequence of this error in Theorem 1, the optimal density function $\phi_{0}$ is incorrectly given in (4.1). Instead, (4.1) should read $\phi_{0}(x)=|v(x)|^{4 /(p+2)} / \int_{G}|v(y)|^{4 /(p+2)} d y$ and the last displayed equation on page 2002 should read $\int_{G} v(x)^{2} d x /$ $\left\{\int_{G}|v(x)|^{4 /(p+2)} d x\right\}^{(p+2) / 2}$. Finally, (3.1) should read

$$
m_{J}=\left\lfloor w_{n}\left\{\frac{n}{r_{J}} \int_{R_{J}} \phi(x) d x\right\}^{1 / 2}\right\rfloor .
$$

I thank Klaus Ritter for pointing out these errors. 